\title{
Biological clock- our 24 hours' rhythms
}

\section{B H N Yasmeen ${ }^{1}$}

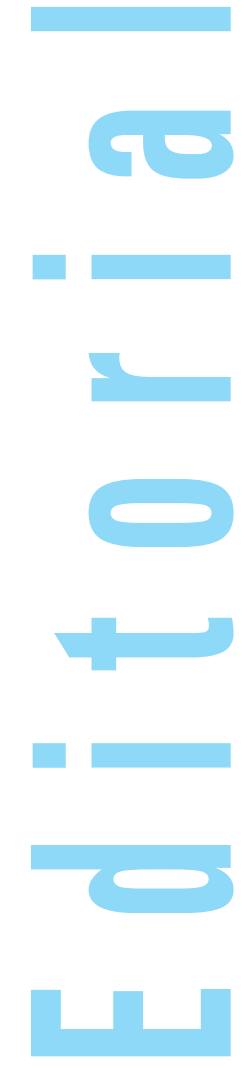

${ }^{1}$ Prof. Dr. B H Nazma Yasmeen MBBS, MCPS, MD (Paed) Editor Northern International Medical College Journal

Professor and Head Dept. of Paediatrics Northern International Medical College, Dhaka e-mail :

prof.nazma.yasmeen@gmail.com

When in 2017, the Nobel Prize in Physiology (or Medicine) was awarded to three U.S. biologists for their research into the molecular mechanism that drives circadian rhythm. ${ }^{1}$ Then the scientists, doctors, chronobiology experts, all are exhibiting their interests on this subject.

If we look behind, in the sixteenth century JeanJacques d'Ortous de Mairan when observed a plant opening and closing its leaflets each day in light and complete darkness, revealing the existence of a biological clock. ${ }^{2,3}$ After that during 18th century, researchers discovered that circadian rhythms synchronize to light-dark cycles and the circadian rhythms drive by our biological clocks. ${ }^{4,5}$ Later on researchers isolated a gene that controls the daily biological rhythm while interacting with the 24-hour light/dark cycle, revealing how the biological clock system works on a molecular level. 6

When we sleep and wake in every 24 hours and other daily patterns of body functions (eating habits, digestion etc.) are part of our Circadian (Latin word meaning "about a day") rhythms, is controlled by the part of the brain that responds to light.7 Neuroscientists suspect that almost every cell in the body contains a biological or circadian clock. These gene-operated clocks are found in different parts of the body such as liver, lungs, connective tissue and muscles. ${ }^{8} \mathrm{~A}$ master clock in the brain coordinates all the body clocks so that they are in synch. ${ }^{9}$ This master clock or main biological clock is located in the suprachiasmatic nuclei (SCNs). The SCN is a group of about 20,000 neurons, located in the hypothalamus. ${ }^{10}$ It also contains receptors for melatonin (sleep hormone). The light arriving to the eyes signals the SCN to reset the sleep wake cycle to a 24 hours one.

This influence is mediated by Melatonin, which triggers drowsiness and promotes sleep. This hormone is produced in the dark (when the sun sets and there is less light) and its secretion is inhibited by light. ${ }^{11}$
Circadian rhythms not only determine sleep patterns, but they also influence hormone release, eating habits and digestion, body temperature, and other important biological functions. ${ }^{12,13}$ According to the National Sleep Foundation, USA during the restorative stage of sleep, blood pressure drops, breathing slows down, blood flow moves to the muscles and tissue is repaired. Several hormones, specially the human growth hormone, are also secreted during sleep. ${ }^{14}$

Twenty-four hours in a day circadian rhythm is active, it never shuts off, therefore its effects are felt at all hours. But it doesn't remain the same from birth to old age. Depending on age and lifestyle, circadian rhythm may fluctuate. A study done in 2015 found that clock-controlled genes fluctuate in the brain as people grow older. ${ }^{15}$ Researchers found that younger brains had a daily rhythm in their clock genes while the rhythm had been lost in many of the genes of older people.

Circadian rhythm acts well in conjunction with healthy sleep habits, such as going to bed and waking at the same time each day. Regular sleep habits are more likely to produce consistent energy levels throughout the day, as well as lower the levels of day time sleepiness. Several researchers also suggest that a healthy circadian rhythm may prevent age-related diseases like dementia, hypertension and Parkinson's disease. ${ }^{16}$

Sleep deprivation or irregular sleep and wake times may disrupt the circadian rhythm, causing various health problems like depression, Seasonal Affective Disorder (SAD), chronic diseases, and jet lag etc. ${ }^{12} \mathrm{~A}$ disrupted sleep pattern also can increase hunger (decreased concentrations of leptin) leading to weight gain, obesity, and other metabolic problems. ${ }^{17}$ Some neuroscientists suggest that repeated psychological stress on the body can disturb the circadian rhythm. ${ }^{18,19}$ 


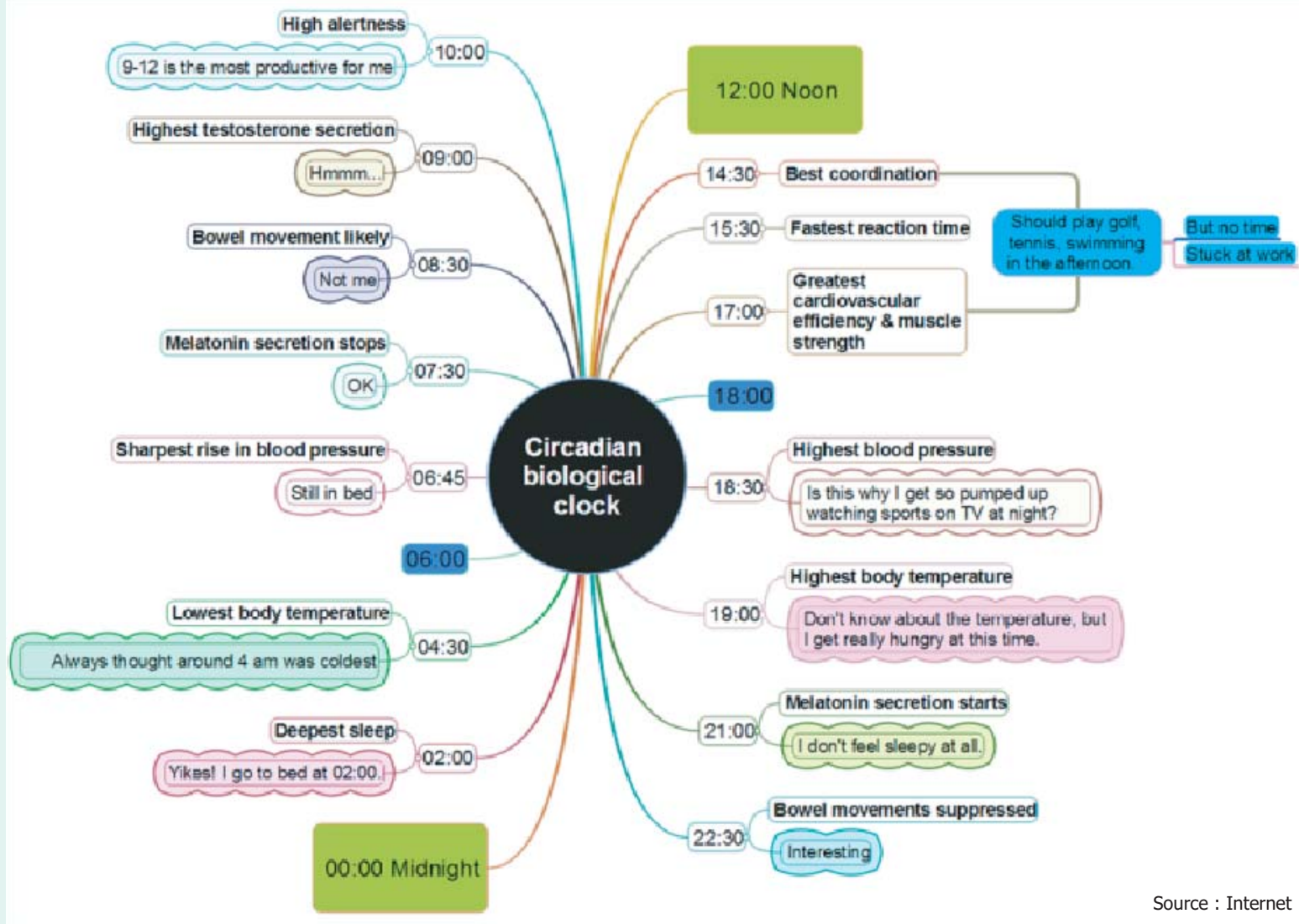

While some circadian rhythm disorders are intrinsic, means a person's body clock is off-kilter from the rest of society's e.g. Delayed sleep phase syndrome (DSPS), Advanced Sleep Phase Disorder (ASPD) etc. Others are circumstantial, or extrinsic, meaning a person's circadian rhythm is out of sync with typical light/darkness patterns. ${ }^{20}$ This is often due to non-traditional work hours, traveling, or exposure to large amounts of artificial light e.g. Shift-work sleep disorder (SWSD), Jet lag etc.

\section{Delayed sleep phase syndrome (DSPS)}

DSPS is adisorder in which a person's sleep is delayed by 2 or more hours beyond the socially acceptable bedtime. This delay in falling asleep causes difficulty in waking up at the desired time in the morning. ${ }^{21}$ For instance, instead of falling asleep at 10:30 pm and waking at 6:30 am, someone will DSPS will fall asleep closer to 1:00 am and have a difficult in waking up in time for school.

DSPS is most common among adolescents; about $16 \%$ of whom have the disorder. ${ }^{22}$ The sufferers often feel extremely tired during the day, experience insomnia, and having a greater risk of depression or other psychiatric problems, including behavioral issues. ${ }^{23}$

\section{Advanced Sleep Phase Disorder (ASPD)}

ASPD is a condition that is characterized by a recurrent pattern of early evening (6:00 pm instead of am 9:00 pm) sleepiness and early morning (2:00 am instead of 5:00 am) awakening. This sleep phase advancement can interfere with daily social and work schedules and results in shortened sleep duration and excessive daytime sleepiness. ${ }^{24}$

Till today very few treatment of Circadian Rhythm Disorders are practiced, e.g

\section{Shift-work sleep disorder (SWSD)}

SWSD results from a conflict between a sufferer's circadian rhythm and work schedule. This schedule may run counter to the internal body clock, leading to insomnia, excessive daytime sleepiness. The excessive sleepiness appears when the individual has to be productive, awake and alert. 25,26

\section{Jet lag}

Jet lag occurs when travelers suffer from disrupted circadian rhythms. When a traveler passes through different time zones, his body's clock will be different from his wristwatch. For example, if he flies in an airplane from Dhaka to New York, he loses 12 hours of time. So when he wakes up at 8:00 a.m., his 
body still thinks it's 8p.m., making him feel groggy and disoriented. His body's clock will eventually reset itself, but this often takes a few days. ${ }^{14,27}$

- Timed exposure to bright light: may help to reset the circadian clock in case of severe circadian rhythm disorders. 28

- Behavior therapy: is commonly used for less severe disorders like DSPS. This therapy include establishing regular sleep-wake times, avoiding naps, sticking to regular morning or afternoon exercise, and avoiding caffeine and bright light from screens a few hours before bedtime.

- Melatonin: has also shown to be an effective treatment for circadian rhythm sleep disorders, especially for jet lag. ${ }^{29}$

- Practice meditation, journaling, breathing techniques, or therapy to reduce stress also help to make a heathy circadian rhythm.

Heathy circadian rhythm means healthy habits. As it helps to promote energy and well-being while lowering the risk of developing certain illnesses and also help them to practice a good habit to make a healthy circadian rhythm.

At this moment, a big question stands in front of us whether our new generation care their biological clock? Can maintain a healthy circadian rhythm? It's true most of them have so much inconsistent sleep schedule that their Melatonin hormone even can't get chance to be secreted. Day by day their life style set them far away from a healthy circadian rhythm. Therefore, we should give our attention to this issue and encourage them to maintained a healthy circadian rhythm by maintained a consistent bed and wake times, limit exposure to artificial light from screens at night, and stick to a consistent eating and exercise routine every day. Also encourage them to practice meditation, journaling, breathing therapy to reduce stress.

\section{Happy new year 2019}

As the new year arrives, at the auspicious moment, I would like to express my warm and heartfelt wishes to all of our readers, authors, reviewers, supporters, advisers and Chief Patron, Honorable Chairman, Northern International Medical College, Prof. Abu Yousuf Md. Abdullah.

Already we have completed our colorful journey of one decade. I am grateful to all of you for your continued support to make our journey smooth and fruitful.

Best wishes for a happy, healthy and successful new year.

\section{References}

1. The Nobel Prize in Physiology or Medicine 2017. www.nobelprize.org. Retrieved 2017-10-06.

2. Sollars P, Pickard G. The Neurobiology of Circadian Rhythms. Psychiatric Clinics of North America, Dec 2015

3. Isaac E. Circadian rhythms in a nutshell. Physiol Genomics. 2000; 3:59-69.
[PubMed]

4. Antoch MP, Song EJ, et al. Functional identification of the mouse circadian Clock gene by transgenic BAC rescue. Cell, May 16, 1997

5. Spoelstra $\mathrm{K}$, Wikelski $\mathrm{M}$, et al. Natural selection against a circadian clock gene mutation in mice. Proceedings of the National Academy of Sciences of the United States of America, Jan 192016

6. Klarsfeld $A$, Birman $S$, et al. Nobel time for the circadian clock. Medecine sciences, June 13, 2017

7. LeGates T.A., Fernandez D.C., et al. Light as a central modulator of circadian rhythms, sleep and affect. Nature Reviews Neuroscience, Jun 11, 2014 Public Health, Aug 2018

8. Dudek $M$ and Meng Q. Running on time: the role of circadian clocks in the musculoskeletal system. Biochemical Journal, Sep 8. 2014

9. Mohawk J, Green C, et al. Central and peripheral circadian clocks in mammals. Annual Review of Neuroscience, Jul 14, 2013

10. Buhr E and Van Gelder R. The Making of the Master Clock. eLife, Aug 20, 2014

11. Pevet $P$, Challet E. Melatonin: both master clock output and internal timegiver in the circadian clocks network. Journal of Physiology (Paris), Dec 2011

12. Farhud, D. Circadian Rhythm, Lifestyle and Health: A Narrative Review. Iranian Journal of Public Health, Aug 2018

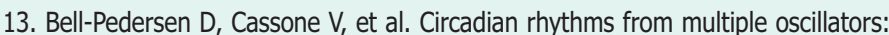
lessons from diverse organisms. Nature Reviews Genetics,July 2015

14. HYPERLINK "https://earthsky.org/team/eleanorimster" lo "Posts by Eleanor Imster" Eleanor Imster. earthsky.org > human-world > cool-facts-aboutyour-biological-clockNovember 3, 2014

15. Chen $C$, Logan $R$, et al. Effects of aging on circadian patterns of gene expression in the human prefrontal cortex. Proceedings of the National Academy of Sciences of the United States of America, Jan 5, 2016

16. Hood S and Amir S. The aging clock: circadian rhythms and later life. Journal of Clinical Investigation, Feb 12017

17. Scheer FA, Hilton MF. Adverse metabolic and cardiovascular consequences of circadian misalignment. Proceedings of the National Academy of Sciences of the United States of America, Mar 17, 2009

18. Helfrich $\square$ Förster $C$, Interactions between psychosocial stress and the circadian endogenous clock. Psych Journal, Dec 26, 2017

19. Chung S, Hoon Son G, et al. Circadian rhythm of adrenal glucocorticoid: Its regulation and clinical implications. Biochimica et BiophysicaActa, May 2011

20. Zhu L and Zee P. Phyllis C. Zee. Circadian Rhythm Sleep Disorders. Neurologic Clinics, Nov 1, 2013

21. Hirshkowitz. Neuropsychiatric Aspects of Sleep and Sleep Disorders " In Yudofsky SC, Hales RE (eds. 2004). Essentials of neuropsychiatry and clinical neurosciences (4th ed. 324-325). Arlington, Virginia: American Psychiatric Publishing. .ISBN 978-1-58562-005-0.

22. Gradisar M and Crowley S. Delayed Sleep Phase Disorder in Youth. Current Opinion in Psychiatry. Aug 25, 2014

23. Figueiro M. Delayed sleep phase disorder: clinical perspective with a focus on light therapy. Nature and Science of Sleep, Apr 62016

24. Dodson, EhrenR, Zee, Phyllis C. "Therapeutics for Circadian Rhythm Sleep Disorders". Sleep Medicine Clinics. 2010; 5 (4): 701-715. doi:10.1016/j.jsmc.2010.08.001. ISSN 1556-407X. PMC 3020104. PMID 21243069.

25. Shift Work Disorder Symptoms | National Sleep Foundation" www. sleepfoundation.org. Retrieved 2019-06-20.

26. Roth, T. Shift work disorder: overview and diagnosis. The Journal of Clinical Psychiatry, Mar 2012

27. "Highlights of Changes from DSM-IV-TR to DSM5"(PDF). American Psychiatric Association. May 17, 2013. Archived from the original(PDF) on September 17, 2013. Retrieved May 23, 2013.

28. Dodson E and Zee P. Therapeutics for Circadian Rhythm Sleep Disorders. Sleep Medicine Clinics, Dec 1, 2011

29. Auger Rm Burgess $\mathrm{H}$, et al. Clinical Practice Guideline for the Treatment of Intrinsic Circadian Rhythm Sleep-Wake Disorders. Journal of Clinical Sleep Medicine, 2015 